\title{
Endoscopic Examination for Patients with Diarrhea after Allogeneic Stem Cell Transplantation
}

\author{
Junichi Kitagawa', Takuro Matsumoto', Yuhei Shibata', Nobuhiko Nakamura1, \\ Hiroshi Nakamura', Soranobu Ninomiya ${ }^{1}$, Yasuhito Nannya ${ }^{1}$, Masahito Shimizu${ }^{2}$, \\ Takeshi Hara' ${ }^{1}$, Hiroshi Araki², Hisashi Tsurumi ${ }^{1}$ \\ ${ }^{1}$ Department of Hematology, Graduate School of Medicine, Gifu University, Gifu, Japan \\ ${ }^{2}$ Department of Gastroenterology, Graduate School of Medicine, Gifu University, Gifu, Japan \\ Email:htsuru@gifu-u.ac.jp
}

How to cite this paper: Kitagawa, J., Matsumoto, T., Shibata, Y., Nakamura, N., Nakamura, H., Ninomiya, S., Nannya, Y., Shimizu, M., Hara, T., Araki, H. and Tsurumi, H. (2017) Endoscopic Examination for Patients with Diarrhea after Allogeneic Stem Cell Transplantation. Open Journal of Internal Medicine, 7, 105-114.

https://doi.org/10.4236/ojim.2017.74011

Received: June 28, 2017

Accepted: October 31, 2017

Published: November 3, 2017

Copyright $\odot 2017$ by authors and Scientific Research Publishing Inc. This work is licensed under the Creative Commons Attribution International License (CC BY 4.0).

http://creativecommons.org/licenses/by/4.0/

\begin{abstract}
Objective: Intestinal graft-versus-host disease (GVHD) represents one of the most serious complications of allogeneic stem cell transplantation (allo-SCT). Endoscopic and histological proof is required due to the number of differential diagnoses manifesting as diarrhea. We investigated the safety of endoscopic biopsies, and the role of conducting biopsies and inspections of the terminal ileum. Patients: Thirty two colonoscopic examinations and 29 biopsies were performed for 19 patients after allo-SCT in our institute between October 2011 and May 2015. Results: Endoscopic examinations and biopsies were performed safely under the policy of transfusing platelets for thrombocytopenia $\left(<30 \times 10^{3} / \mu \mathrm{L}\right)$. For biopsied cases, the diagnostic consistency rate with endoscopic findings was $60 \%$, with a tendency toward negative correlations with early examinations after diarrhea onset ( $25 \%$ for $0-1$ days; $62.5 \%$ for later) or low-grade GVHD according to Freiburg criteria (41.2\% for grade 1, $66.7 \%$ for grade 2, $100 \%$ for higher). The terminal ileum was inspected with colonoscopy in 13 cases. Endoscopic diagnoses of the ileum were provided in 11 cases and histological diagnoses in 9 cases. Diagnostic consistency for diagnosis of the terminal ileum between endoscopy and histology was 77.8\%. Conclusion: Because endoscopic and histopathological findings do not always match, caution is required when focusing on endoscopic findings alone, as there is a risk of misdiagnosis. Extensive inspection of the terminal ileum with biopsy appears useful to identify otherwise undetected lesions. Our data thus support invasive endoscopic examinations for gastrointestinal complications, including ileac inspection and biopsies under appropriate management.
\end{abstract}




\section{Keywords}

Intestinal GVHD, Endoscopic Evaluation, Ileoscopy, Colonoscopy

\section{Introduction}

Allogeneic stem cell transplantation (allo-SCT) has become an essential modality for intractable hematological diseases. Common complications of allo-SCT include infections, conditioning regimen-related toxicities, and graft-versus-host disease (GVHD). Acute GVHD mainly affects the skin, liver and gastrointestinal (GI) tract [1] [2] [3] [4]. The GI tract is commonly involved in acute GVHD along with the skin and liver, and is affected in up to $50 \%$ of patients who develop acute GVHD [5]. Severe acute GVHD is associated with considerable morbidity and mortality.

Diarrhea is a common and complicating symptom after allo-SCT. This symptom can result from an extremely wide range of etiologies, including preparative regimen-related toxicity, infection, and GVHD [6] [7], underscoring the significance of making a definite diagnosis of the cause of diarrhea in the management of allo-SCT. Along with microbiological investigations as the first step in the diagnostic work-up for post-allo-SCT patients with diarrhea, microscopic examinations and biopsy of affected lesions also constitute an essential step. However, practical application of microscopic biopsy entails several concerns. One is the invasiveness, particularly for post-SCT patients with thrombocytopenia, in which the risk of hemorrhagic complications discourages physicians. Other concerns are the interpretation of inconsistent results between endoscopic and pathological findings, and the unestablished role of sampling from multiple sites including the terminal ileum. The present study investigated the safety of endoscopic biopsies, and the role of conducting biopsies and inspections of the terminal ileum.

\section{Patients and Methods}

\subsection{Patients}

Among the 46 patients who received allo-SCT in our institute between October 2011 and May 2015, 32 colonoscopic examinations were performed for 19 patients. This was a retrospective study that was approved by the Institutional Review Board at Gifu University Hospital and informed consent was obtained from each patient. Myeloablative conditioning (MAC) regimens comprised cyclophosphamide (CY, $120 \mathrm{mg} / \mathrm{kg}$ ) in combination with either $12 \mathrm{~Gy}$ of total body irradiation (TBI) or busulfan (BU, $12.8 \mathrm{mg} / \mathrm{kg}$ for intravenous form) with or without cytarabine (Ara-C). Reduced-intensity conditioning (RIC) regimens consisted of CY (60 mg/kg), fludarabine (Flu, $150 \mathrm{mg} / \mathrm{kg}$ ) and $2-4$ Gy of TBI with or without Ara-C $(n=6)$. Microbiological analyses of stools, including bacterial cultures and the Clostridium difficile toxin assay, were also performed in parallel with 
colonoscopy. Cytomegalovirus (CMV) infection was monitored using a pp 65 antigenemia assay once weekly. CMV antigenemia was described in terms of the number of positive cells per slide using the C10/11 method (150,000 cells applied) [8].

\subsection{Colonoscopy and Procedures}

All colonoscopies were performed using an Olympus colonoscope (CF-H260I, CF-Q260I, PCF-Q260AI, CF-HQ-290I, CF-H290I, or PCF-290I; Olympus Optical, Tokyo, Japan). Colonoscopic procedures were performed with conscious sedation using diazepam or midazolam. Biopsies were obtained using standard endoscopic forceps. Platelet transfusions were administered before colonoscopies at the discretion of the hematologist when platelet counts were below 30,000/ $\mu \mathrm{L}$. Endoscopists determined the preparation for colonoscopy, route of intubation and indications for biopsy. Endoscopic findings were graded using the Freiburg criteria, summarized as: Grade 1, no findings compatible with upper grades; Grade 2, spotted erythema, initial aphthous lesions; Grade 3, aphthous lesions (Crohn-like) or focal erosions; or Grade 4, confluent defects, ulcerations, complete denudation of the mucosa [9]. GI stages of acute GVHD were defined as follows: Stage 0, $<500 \mathrm{~mL} /$ day of diarrhea; Stage 1, $500-999 \mathrm{~mL}$ /day of diarrhea; Stage 2, 1000 $1499 \mathrm{~mL} /$ day of diarrhea; Stage 3, $\geq 1500 \mathrm{~mL} /$ day of diarrhea; Stage 4, severe abdominal pain with or without ileus [10]. Biopsies were stained using hematoxylin and eosin, and evaluated by two pathologists. The histologic diagnosis of GI GVHD was based on the identification of apoptotic epithelial cells that were usually found in the regenerative or stem-cell areas (Figure 1). CMV infection of mucosa was judged by staining for CMV antigen.

\section{Results}

\subsection{Patient Characteristics}

Characteristics of patients and allo-SCT procedures are summarized in Table 1. GVHD prophylaxis included cyclosporine (CsA) or tacrolimus (TAC) from day-1, combined with short-term methotrexate (MTX).

\subsection{Colonoscopy}

Thirty colonoscopic examinations were performed for 19 patients in search of etiologies for diarrhea after allo-SCT. Seven patients received two or more colonoscopic examinations. The reasons of repeated examinations were sustained diarrhea in 5 and relapse of diarrhea in 2. Volume of diarrhea before colonoscopy was $<500 \mathrm{mlL} /$ day in 10 cases, $500-999 \mathrm{~mL} /$ day in 13,1000 to $1499 \mathrm{~mL} /$ day in 3 , 1500 to $1999 \mathrm{~mL} /$ day in 3, and $\geq 2000 \mathrm{~mL} /$ day in 3. Polyethylene glycol and glycerin enema were used for bowel preparations in 10 and 3 cases, respectively, and in 19 case no preparation was done. Colonoscopic biopsy was performed in 29 examinations for 19 patients. Median duration from transplantation to first colonoscopy was 36 days (range, 15 - 79 days), showing no significant difference 


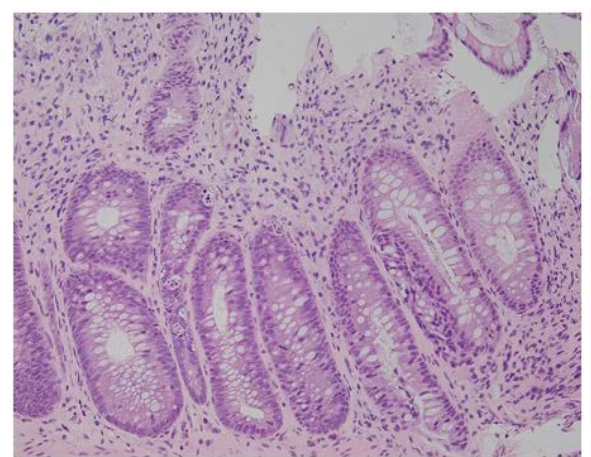

(a)

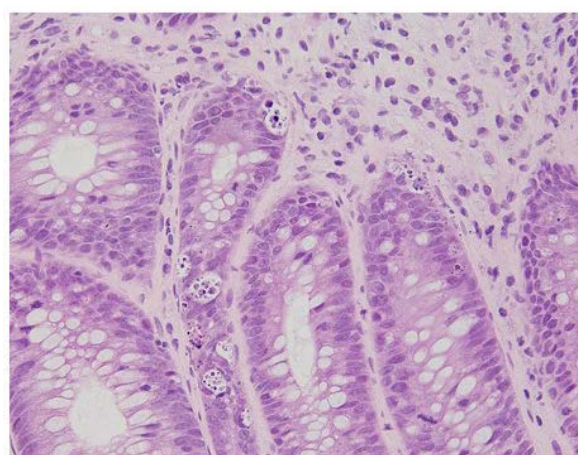

(b)

Figure 1. Photomicrograph of biopsied specimen of lower alimentary tract (OR rectum). Crypts were decreased with lymphocytic infiltration beyond basement membrane as well as abundant apoptotic change of epithelial cells. Also, interstitial edema and fibrosis were evident. These findings strongly suggested GVHD.

Table 1. Patients' characteristics $(n=19)$.

\begin{tabular}{|c|c|c|}
\hline & $\mathrm{n}$ & $\%$ \\
\hline \multicolumn{3}{|c|}{ Age median (range) } \\
\hline & 39 & $(16-65)$ \\
\hline \multicolumn{3}{|l|}{ Sex } \\
\hline Male/female & $11 / 8$ & \\
\hline \multicolumn{3}{|l|}{ Diagnosis } \\
\hline AML & 6 & 31.6 \\
\hline ALL & 3 & 15.8 \\
\hline MDS & 3 & 15.8 \\
\hline CML & 1 & 5.3 \\
\hline $\mathrm{AA}$ & 1 & 5.3 \\
\hline ML & 5 & 26.3 \\
\hline \multicolumn{3}{|l|}{ Stem cell source } \\
\hline PBSC & 5 & 26.3 \\
\hline $\mathrm{BM}$ & 7 & 36.8 \\
\hline $\mathrm{CB}$ & 7 & 36.8 \\
\hline \multicolumn{3}{|c|}{ Conditioning regimen } \\
\hline MAC & 11 & 57.9 \\
\hline RIC & 8 & 42.1 \\
\hline \multicolumn{3}{|c|}{ GVHD prophylaxis } \\
\hline CsA + MTX & 9 & 47.4 \\
\hline TAC + MTX & 10 & 52.6 \\
\hline \multicolumn{3}{|c|}{ GI stage of acute GVHD at colonoscopy $(\mathrm{n}=32)$} \\
\hline 0 & 12 & 37.5 \\
\hline 1 & 13 & 40.6 \\
\hline 2 & 1 & 3.1 \\
\hline 3 & 3 & 9.4 \\
\hline 4 & 3 & 9.4 \\
\hline
\end{tabular}

AML: acute myelogenous leukemia; ALL: acute lymphoid leukemia; MDS: myelodysplasitc syndromes; CML: chronic myelogenous leukemia; AA: aplastic anemia; ML: malignant lymphoma; BM: bone marrow; PBSC: peripheral blood stem cell; CB: cord blood; MAC: myeloablative conditioning regimen; RIC: reduced-intensity conditioning regimen; CsA: cyclosporine; MTX: methotrexate; TAC: tacrolimus; GI: gastrointestinal; GVHD: graft-versus-host disease. 
from the MAC and RIC groups (41 days; $p=0.77$ ). Median duration from onset of diarrhea to colonoscopy was 2 days (range, 0 - 9 days). Median platelet count at biopsy was $40 \times 10^{3} / \mu \mathrm{L}$ (range, $10 \times 10^{3} / \mu \mathrm{L}-309 \times 10^{3} / \mu \mathrm{L}$ ). Platelet transfusion was performed in 11 cases before colonoscopy, with median platelet counts of $26 \times 10^{3} / \mu \mathrm{L}$ (range, $10 \times 10^{3} / \mu \mathrm{L}-40 \times 10^{3} / \mu \mathrm{L}$ ) at the time of biopsy and $27 \times$ $10^{3} / \mu \mathrm{L}$ (range, $14 \times 10^{3} / \mu \mathrm{L}-65 \times 10^{3} / \mu \mathrm{L}$ )the next day. No major bleeding events were observed after these procedures.

\subsection{Comparison of Endoscopic and Histological Diagnoses}

Endoscopic diagnoses were GVHD, CMV, and thrombotic microangiopathy (TMA) in 25, 4, and 1 cases, respectively. Endoscopic grade of GVHD was 1, 2, 3, or 4 in $11,8,4$, and 2 cases, respectively. The cases diagnosed as having Grade 1 presented edematous or tortoise-shell pattern only. Abnormal colonic mucosa was observed in 30 cases, and biopsy was performed in 29 cases. For those 29 cases, we compared colonoscopic diagnosis and pathological findings (Table 2). Histological evaluation in colonoscopically diagnosed GVHD cases revealed GVHD in 11 , GVHD concomitant with CMV colitis in 3, CMV colitis in 1, and unspecific or colitis/proctitis in the other 7 cases. The median number of biopsies were 3 (range, 1 - 7), and in 12 of the 14 patients who were histologically diagnosed with GVHD, all biopsy samples showed features of GVHD. The diagnostic accuracy of GVHD between endoscopy and histology was 60\%. A trend was seen towards higher consistency between endoscopic and histological diagnoses in cases with high endoscopic grades (Table 3). In addition, a short interval from onset of diarrhea to endoscopy was associated with a low rate of diagnosis accuracy (Table 3). Microbiological analyses of stools were negative in all biopsy cases. CMV antigenemia was positive in all cases of histologically confirmed CMV colitis (median, 14 cells per 150,000 granulocytes applied for C10/11; range, 5 - 48 cells). In one endoscopically diagnosed case of TMA, the histology supported the endoscopic diagnosis.

\subsection{Ileoscopic Diagnosis}

The terminal ileum was inspected with colonoscopy in 13 cases (Table 4). Endoscopic diagnoses of the ileum were provided in 11 cases and histological diagnoses in 9 cases. Diagnostic consistency for diagnosis of the terminal ileum between endoscopy and histology was $77.8 \%$ (7 of 9 cases). With ileoscopy, we were able to histologically diagnose one case of GVHD (Case 6) and one case of TMA (Case 10) as cases in which colonoscopy revealed no findings (Figure 2). On the other hand, two cases with histologically confirmed GVHD in the colon (Cases 4 and 8) showed no findings on ileoscopy. These two cases (Cases 4 and 8) might be performed colonoscopy before extension of the ileum because both of them had stage 1 GVHD. The histological diagnoses of the terminal ileum and colon differed in 1 case (Case 5). 
Table 2. Endoscopic and histological finding.

\begin{tabular}{cccccc}
\hline Endoscopic diagnosis & & Performed biopsy & Histological finding & $\mathrm{n}$ & $\%$ \\
\hline GVHD & 25 & 21 & GVHD & 10 & 47.6 \\
& & & GVHD + CMV & 3 & 14.3 \\
& & & CMV & 1 & 4.8 \\
CMV & 4 & 4 & colitis/proctitis & 7 & 33.3 \\
& & & CMV & 3 & 75.0 \\
TMA & 1 & 1 & GVHD & 1 & 25.0 \\
colitis/proctitis & 2 & 2 & TMA & 1 & 100 \\
\hline
\end{tabular}

GVHD: graft-versus-host disease; CMV: Cytomegalovirus; TMA: thrombotic microangiopathy.

Table 3. Diagnostic accuracy of GVHD.

\begin{tabular}{cccc}
\hline Endoscopic grade & (n) & Histological GVHD diagnosis (n) & Consistency rate (\%) \\
\hline 1 & 12 & 5 & 41.2 \\
2 & 6 & 4 & 66.7 \\
3 & 3 & 3 & 100 \\
4 & 1 & 1 & 100 \\
after onset of diarrhea & $(\mathrm{n})$ & & 25 \\
day 0, 1 & 4 & 1 & 100 \\
day 2, 3 & 4 & 4 & 61.5 \\
$\geq$ day 4 & 13 & 8 & \\
\hline
\end{tabular}

GVHD: graft-versus-host disease.

Table 4. Comparison between ileoscopic and colonocsopic findings and diagnoses.

\begin{tabular}{ccccc}
\hline & \multicolumn{2}{c}{ Ileoscopic finding } & \multicolumn{2}{c}{ Colonoscopic finding } \\
\cline { 2 - 5 } & Ileoscopic diagnosis Histological diagnosis & Colonoscopic diagnosis & Histological diagnosis \\
\hline 1 & GVHD & GVHD & GVHD & GVHD \\
2 & CMV & CMV & CMV & CMV \\
3 & CMV & CMV & CMV & CMV \\
4 & No finding & None & GVHD & GVHD \\
5 & GVHD & CMV & GVHD & GVHD \\
6 & CMV & GVHD & No finding & None \\
7 & GVHD & None & GVHD & None \\
8 & No finding & None & CMV & GVHD \\
9 & GVHD & GVHD & GVHD & GVHD \\
10 & TMA & TMA & No finding & No finding \\
11 & GVHD & None & GVHD & colitis \\
\hline
\end{tabular}

GVHD: graft-versus-host disease; CMV: Cytomegalovirus; TMA: thrombotic microangiopathy. 


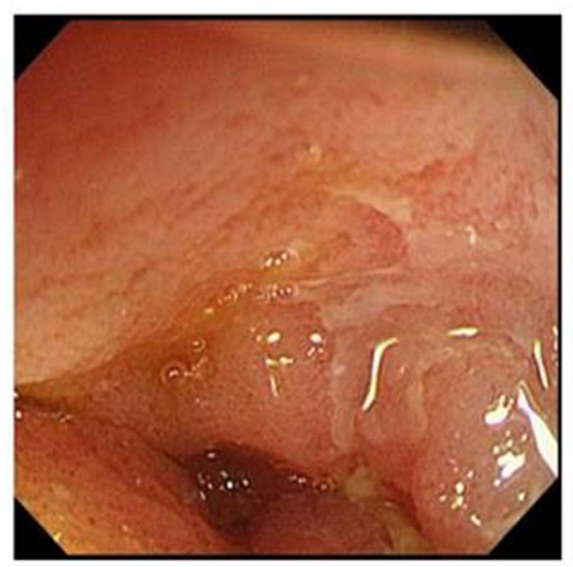

$(\mathrm{a}-1)$

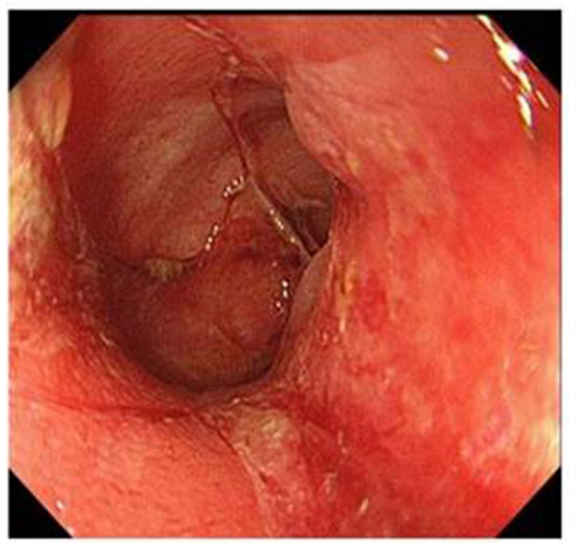

(b-1)

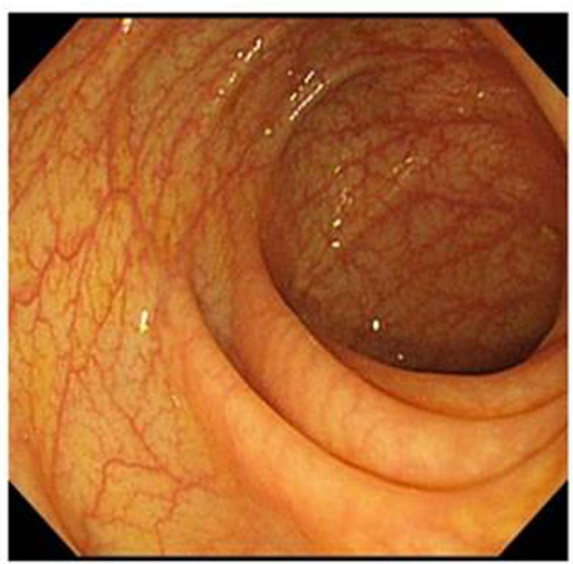

$(\mathrm{a}-2)$

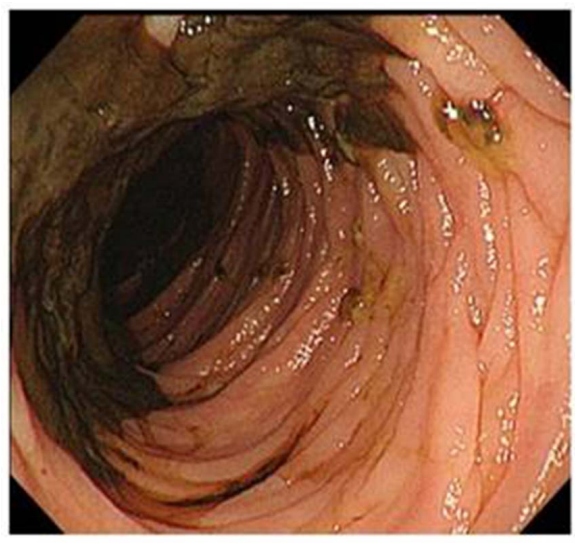

(b-2)

Figure 2. (a) A case of GVHD in the terminal ileum. Geographical ulcer in the terminal ileum only (a-1); with normal appearance of the colon (a-2); (b) A case of TMA in the terminal ileum. Confluent defects, ulcerations and complete denudation of the mucosa in the terminal ileum only (b-1); with normal mucosa in the colon (b-2).

\section{Discussion}

Intestinal GVHD represents one of the most serious complications of allo-SCT, and endoscopic and, where possible, histological proof of GVHD is desirable due to the number of differential diagnoses after allo-SCT that can manifest as diarrhea. In particular, distinguishing GVHD from infectious etiologies is essential because of the reciprocal indication of immunosuppression. However, low platelet counts just after engraftment often cause concerns about the safety of endoscopic procedures, especially when selecting specimens for removal from the fragile mucosa. At our institute, we provided the platelet transfusion before endoscopic procedures when the platelet count was below $30 \times 10^{3} / \mu \mathrm{L}$ and no hemorrhagic complications were observed. Our policy might be overly cautious, because Inamoto et al. reported that no complications developed with platelet transfusion for 30 patients, although platelet count at biopsy was less than $20 \times 10^{3} / \mu \mathrm{L}$ in 12 patients [11]. However, we apply a more invasive approach including inspection 
of the terminal ileum and procurement of specimens deep in the mucosa, so distinct safety margins are warranted and demonstration that our criteria are safe even for these invasive procedures is of note.

The endoscopic findings for intestinal GVHD range from almost normal to extensive edema and mucosal sloughing. Whether these findings can predict the histological diagnosis and endoscopic grade of GVHD remains controversial. Kreisel et al. evaluated 175 consecutive patients with intestinal GVHD examined both endoscopically and histologically, and reported high diagnostic accuracy. Setting a cutoff between endoscopic grades 1 and 2, endoscopy offered 89.2\% sensitivity, $79.4 \%$ specificity, a positive-predictive value of $79.6 \%$ and a negative-predictive value of $89.0 \%$ [9]. This is consistent with our result that high endoscopic GVHD grade was associated with high consistency with histology. However, the overall consistency rate between endoscopy and histology in our series was lower compared with that reported by Kreisel et al. We attributed this to the different timings for conducting endoscopic examinations. In concrete terms, Kreisel et al. performed endoscopies between 21 and 405 days after allo-SCT, and before 100 days in 65\%, after 100 days in 35\% [9]. Martinez et al. reported performing first endoscopy at a median of 57 days (range, 16 - 155 days) after allo-SCT [12]. The median timing of first endoscopy in our study was 36 days (range, 15 - 79 days), substantially earlier than in those reports. Martinez et al. also explained the discrepancies between these studies by differences in the timings of endoscopic examination [12]. We performed endoscopy immediately when intestinal acute GVHD was suspected due to diarrhea after engraftment. In fact, most of GI stage of acute GVHD was low, and the same results were seen for endoscopic grade. Moreover, the diagnostic accuracy of GVHD was much lower when endoscopy was performed within 1 day of diarrhea onset. Although endoscopy for the treatment of GI GVHD should be performed early, attention must be paid to the evaluation of endoscopic findings performed soon after onset diarrhea.

The fact that endoscopic findings of GVHD are similar to those of infections is important [13] [14]. CMV infection in particular may mimic acute GVHD [9]. Our final evaluation with histological examinations revealed 7 cases (23\%) with CMV infection, with positive results for CMV antigenemia in all of them. If high-level CMV antigenemia was defined as $\geq 10$ positive cells per 150,000 granulocytes applied for C10/11 [8], 83\% (5 cases) were high level at the time of biopsy. On the other hand, antigenemia value and endoscopic finding grade were not correlated (data not shown). We thought that high-level CMV antigenemia might be useful to support the diagnosis CMV-GI disease, but the severity of the endoscopic findings was not reflected.

We showed topical diversity in GI tract lesions after allo-SCT by demonstrating different diagnoses between ileoscopic and colonoscopic examinations in some cases. Ross et al. reported higher accuracy of biopsies in the rectosigmoid colon than in the upper GI tract. Their study analyzed 112 patients who underwent simultaneous endoscopic biopsies of the stomach, duodenum and recto- 
sigmoid colon within the first 100 days after allo-SCT. Rectosigmoid biopsies showed the highest sensitivity (95.6\%) and specificity (100\%) [15]. Kreisel et al. found that in about $20 \%$ of patients with ileo-colonoscopy, typical GVHD lesions could be found only in the terminal ileum [9]. They recommended terminal ileum inspection even if the colon appeared endoscopically normal. In addition, the terminal ileum was the site most commonly affected by TMA [11]. In this study, 2 cases had GVHD lesions only in the ileum, and in another case, the diagnosis in the ileum differed from that of the colon, which underlies the significance of performing inspection of the terminal ileum.

Our data suggest colonoscopy as a safe, effective method to examine for lower GI complications after allo-SCT. Early definitive diagnosis is needed, because GVHD, CMV and TMA each require different therapeutic approaches. Hematologists and endoscopists should not hesitate to perform colonoscopy, if possible, ileal intubation, and biopsy when lower GI complications arise.

\section{Conflict of Interest}

We have no conflicts of interest to declare.

\section{References}

[1] McDonald, G.B., Shulman, H.M., Sullivan, K.M. and Spencer, G.D. (1986) Intestinal and Hepatic Complications of Human Bone Marrow Transplantation. Part I. Gastroenterology, 90, 460-477. https://doi.org/10.1016/0016-5085(86)90949-2

[2] McDonald, G.B., Shulman, H.M., Sullivan, K.M. and Spencer, G.D. (1986) Intestinal and Hepatic Complications of Human Bone Marrow Transplantation. Part II. Gastroenterology, 90, 770-784. https://doi.org/10.1016/0016-5085(86)91137-6

[3] Deeg, H.J. and Antin, J.H. (2006) The Clinical Spectrum of Acute Graft-versus-Host Disease. Seminars in Hematology, 43, 24-31. https://doi.org/10.1053/j.seminhematol.2005.09.003

[4] McDonald, G.B. (2010) Hepatobiliary Complications of Hematopoietic Cell Transplantation, 40 Years on. Hepatology, 51, 1450-1460. https://doi.org/10.1002/hep.23533

[5] Przepiorka, D., Smith, T.L., Folloder, J., Khouri, I., Ueno, N.T., Mehra, R., Körbling, M., Huh, Y.O., Giralt, S., Gajewski, J., Donato, M., Cleary, K., Claxton, D., Braunschweig, I., van Besien, K., Andersson, B.S., Anderlini, P. and Champlin, R. (1999) Risk Factors for Acute Graft-versus-Host Disease after Allogeneic Blood Stem Cell Transplantation. Blood, 94, 1465-1470.

[6] Shen, B., Khan, K., Ikenberry, S.O., Anderson, M.A., Banerjee, S., Baron, T., Ben-Menachem, T., Cash, B.D., Fanelli, R.D., Fisher, L., Fukami, N., Gan, S.I., Harrison, M.E., Jagannath, S., Lee Krinsky, M., Levy, M., Maple, J.T., Lichtenstein, D., Stewart, L., Strohmeyer, L. and Dominitz, J.A. (2010) The Role of Endoscopy in the Management of Patients with Diarrhea. Gastrointestinal Endoscopy, 71, 887-892. https://doi.org/10.1016/j.gie.2009.11.025

[7] Cox, G.J., Matsui, S.M., Lo, R.S., Hinds, M., Bowden, R.A., Hackman, R.C., Meyer, W.G., Mori, M., Tarr, P.I., Oshiro, L.S., et al. (1994) Etiology and Outcome of Diarrhea after Marrow Transplantation: A Prospective Study. Gastroenterology, 107, 1398-1407. https://doi.org/10.1016/0016-5085(94)90542-8 
[8] Mori, T., Okamoto, S., Matsuoka, S., Yajima, T., Wakui, M., Watanabe, R., Ishida, A., Iwao, Y., Mukai, M., Hibi, T. and Ikeda, Y. (2000) Risk-Adapted Pre-Emptive Therapy for Cytomegalovirus Disease in Patients Undergoing Allogeneic Bone Marrow Transplantation. Bone Marrow Transplantation, 25, 765-769. https://doi.org/10.1038/sj.bmt.1702227

[9] Kreisel, W., Dahlberg, M., Bertz, H., Harder, J., Potthoff, K., Deibert, P., Schmitt-Graeff, A. and Finke, J. (2012) Endoscopic Diagnosis of Acute Intestinal GVHD Following Allogeneic Hematopoietic SCT: A Retrospective Analysis in 175 Patients. Bone Marrow Transplant, 47, 430-438.

https://doi.org/10.1038/bmt.2011.137

[10] Irani, J.L., Cutler, C.S., Whang, E.E., Clancy, T.E., Russell, S., Swanson, R.S., Ashley, S.W., Zinner, M.J. and Raut, C.P. (2008) Severeacute Gastrointestinal Graft-vs-Host disease: An Emerging Surgical Dilemma in Contemporary Cancer Care. Archives of Surgery, 143, 1041-1045. https://doi.org/10.1001/archsurg.143.11.1041

[11] Inamoto, Y., Ito, M., Suzuki, R., Nishida, T., Iida, H., Kohno, A., Sawa, M., Murata, M., Nishiwaki, S., Oba, T., Yanada, M., Naoe, T., Ichihashi, R., Fujino, M., Yamaguchi, T., Morishita, Y., Hirabayashi, N., Kodera, Y., Miyamura, K. and Nagoya Blood and Marrow Transplantation Group (2009) Clinicopathological Manifestations and Treatment of Intestinal Transplant-Associated Microangiopathy. Bone Marrow Transplant, 44, 43-49. https://doi.org/10.1038/bmt.2008.419

[12] Martínez, C., Rosales, M., Calvo, X., Cuatrecasas, M., Rodriguez-Carunchio, L., Llach, J., Fernández-Avilés, F., Rosiñol, L., Rovira, M., Carreras, E. and Urbano-Ispízua, A. (2012) Serial Intestinal Endoscopic Examinations of Patients with Persistent Diarrhea after Allo-SCT. Bone Marrow Transplant, 47, 694-699. https://doi.org/10.1038/bmt.2011.143

[13] Snover, D.C. (1985) Mucosal Damage Simulating Acute Graft-versus-Host Reaction in Cytomegalovirus Colitis. Transplantation, 39, 669-670. https://doi.org/10.1097/00007890-198506000-00019

[14] Müller, C.I., Zeiser, R., Grüllich, C., Finke, J., Bertz, H., Schmitt-Gräff, A. and Kreisel, W. (2004) Intestinal Cryptosporidiosis Mimicking Acute Graft-versus-Host Disease Following Matched Unrelated Hematopoietic Stem Cell Transplantation. Transplantation, 77, 1478-1479. https://doi.org/10.1097/01.TP.0000121806.42836.45

[15] Ross, W.A., Ghosh, S., Dekovich, A.A., Liu, S., Ayers, G.D., Cleary, K.R., Lee, J.H. and Couriel, D. (2008) Endoscopic Biopsy Diagnosis of Acute Gastrointestinal Graftversus-Host Disease: Rectosigmoid Biopsies Are More Sensitive Than Upper Gastrointestinal Biopsies. The American Journal of Gastroenterology, 103, 982-989. https://doi.org/10.1111/j.1572-0241.2007.01639.x 